\title{
Diabetic retinopathy is associated with early autonomic dysfunction assessed by exercise-related heart rate changes
}

\author{
C.K. Kramer ${ }^{1}$, C.B. Leitão ${ }^{1}$, M.J. Azevedo ${ }^{1}$, F.B. Valiatti ${ }^{2}$, T.C. Rodrigues ${ }^{1}$, L.H. Canani ${ }^{1}$ \\ and J.L. Gross ${ }^{1}$ \\ ${ }^{1}$ Serviço de Endocrinologia, Hospital de Clínicas de Porto Alegre, Universidade Federal do Rio Grande \\ do Sul, Porto Alegre, RS, Brasil \\ 2Divisão de Retina e Vítreo, Hospital Banco de Olhos de Porto Alegre, Porto Alegre, RS, Brasil \\ Correspondence to: C.K. Kramer, Serviço de Endocrinologia, Hospital de Clínicas de Porto Alegre, \\ UFRGS, Rua Ramiro Barcelos, 2350, Ed. 12, 4 andar, 90035-003 Porto Alegre, RS, Brasil \\ Fax: +55-51-2101-8777. E-mail: ckkramer@terra.com.br
}

\begin{abstract}
Diabetic retinopathy has been associated with cardiac autonomic dysfunction in both type 1 and type 2 diabetes mellitus (DM) patients. Heart rate (HR) changes during exercise testing indicate early alterations in autonomous tonus. The aim of the present study was to investigate the association of diabetic retinopathy with exercise-related HR changes. A cross-sectional study was performed on 72 type 2 and 40 type 1 DM patients. Autonomic dysfunction was assessed by exercise-related HR changes (Bruce protocol). The maximum HR increase, defined as the difference between the peak exercise rate and the resting rate at baseline, and $H R$ recovery, defined as the reduction in $\mathrm{HR}$ from the peak exercise to the HR at 1, 2, and 4 min after the cessation of the exercise, were determined. In type $2 \mathrm{DM}$ patients, lower maximum $\mathrm{HR}$ increase $(\mathrm{OR}=1.62,95 \% \mathrm{Cl}=1.03-2.54 ; \mathrm{P}=0.036)$, lower $\mathrm{HR}$ recovery at $2(\mathrm{OR}=2.04,95 \% \mathrm{Cl}=1.16-3.57 ; \mathrm{P}=0.012)$ and $4 \min (\mathrm{OR}=2.67,95 \% \mathrm{Cl}=1.37-5.20 ; \mathrm{P}=0.004)$ were associated with diabetic retinopathy, adjusted for confounding factors. In type $1 \mathrm{DM}$, the absence of an increase in $\mathrm{HR}$ at intervals of $10 \mathrm{bpm}$ each during exercise added $100 \%$ to the odds for diabetic retinopathy $(\mathrm{OR}=2.01,95 \% \mathrm{Cl}=1.1-3.69 ; \mathrm{P}=0.02)$ when adjusted for DM duration, A1c test and diastolic blood pressure. In conclusion, early autonomic dysfunction was associated with diabetic retinopathy. The recognition of HR changes during exercise can be used to identify a high-risk group for diabetic retinopathy.
\end{abstract}

Key words: Type 2 diabetes mellitus; Type 1 diabetes mellitus; Heart rate changes; Diabetic retinopathy

Research partially supported by PRONEX (\#04/0888-3), CNPq (\#479484-2007-4) and Fundo de Incentivo à Pesquisa (FIPE) do Hospital de Clínicas de Porto Alegre.

Received February 19, 2008. Accepted November 18, 2008

\section{Introduction}

Hypertension is a well-known risk factor for diabetic retinopathy (1). Even minor blood pressure (BP) increases during ambulatory BP monitoring might influence the development of diabetic retinopathy (2), probably due to decreased vasomotor control of the circulation of the retinal vessels allowing even small alterations in BP to impact the retina.

Diabetic retinopathy has been associated with cardiac autonomic dysfunction in both type 1 (3) and type 2 diabe- tes mellitus (DM) patients (4). Heart rate (HR) changes related to exercise testing $(5,6)$ may detect early alterations in autonomous tonus and are a major predictor of sudden death and cardiac mortality in healthy and postmyocardial infarct patients (7-9).

Therefore, the aim of the present study was to investigate the association of diabetic retinopathy with a specific $\mathrm{HR}$ profile related to exercise testing in DM patients in the absence of both ischemic heart disease and clinical autonomic neuropathy. 


\section{Patients and Methods}

\section{Patients}

A cross-sectional study was performed on type 1 and type 2 DM patients diagnosed according to American Diabetes Association criteria (10) and regularly attending the DM outpatient clinic at Hospital de Clínicas de Porto Alegre, Brazil, since 1994. Patients were selected from a cohort of 270 type $2 \mathrm{DM}$ (11) and 349 type 1 DM patients based on the presence of funduscopic evaluation by an ophthalmologist and exercise electrocardiograph testing without evidence of myocardial ischemia. The exclusion criteria were age less than 18 years (type $1 \mathrm{DM}$ ), creatinine $>1.5 \mathrm{mg} / \mathrm{dL}$, other renal diseases, presence of persistent cardiac arrhythmia, abnormal sensitive peripheral tests (Achilles tendon reflexes, vibration or sensory perception tests), autonomic symptoms and orthostatic hypotension. The autonomic symptoms evaluated were chronic diarrhea, syncope or vasomotor symptoms and orthostatic hypotension was defined as systolic BP drop of $\geq 20 \mathrm{mmHg}$ or diastolic $\mathrm{BP} \geq 10 \mathrm{mmHg}$ at $1 \mathrm{~min}$ in the standing position after decubitus.

The study protocol was approved by the Ethics Committee of the hospital, and written informed consent was obtained from all patients.

\section{Clinical evaluation}

Patients underwent an interview and clinical examination to record demographic and anthropometrical data, as previously described $(11,12)$. BP was measured twice on the right arm to the nearest $2 \mathrm{mmHg}$ after a 10-min rest using a standard mercury sphygmomanometer (phases I and $\mathrm{V}$ of Korotkoff sounds) on decubitus. BP was also recorded $1 \mathrm{~min}$ after standing.

Fundus eye examination was performed by an experienced ophthalmologist after mydriasis and diabetic retinopathy was classified using the scale developed by the Global Diabetic Retinopathy Group $(13,14)$. The diabetic retinopathy level was based on the most severe degree of retinopathy in the worst eye affected. For the purpose of this study, patients were grouped according to the presence or absence of any degree of diabetic retinopathy.

\section{Cardiovascular autonomic evaluation}

Exercise electrocardiography was conducted according to the standard Bruce protocol utilizing a computerized database (15). Cardioactive medications were stopped one week before the exam.

Data on symptoms, HR and rhythm, BP and estimated workload in metabolic equivalents (METs) were collected midway through each stage of the exercise protocol, at peak exercise and at 1,2 , and 4 min after cessation of exercise. METs were estimated according to standard tables and are equal to $3.5 \mathrm{~mL}$ of oxygen uptake per kilogram of body weight per minute.

$\mathrm{HR}$ increase was defined as the difference between the peak exercise rate and resting rate at baseline. HR recovery was defined as the reduction in the rate from the peak exercise level to the rate at 1, 2, and $4 \mathrm{~min}$ after cessation of exercise. The BP increase was defined as the difference between the peak exercise BP and resting BP; recovery $\mathrm{BP}$ was defined as the reduction in $\mathrm{mmHg}$ from the peak of exercise level to the $\mathrm{BP}$ at 1,2 , and $4 \mathrm{~min}$ after the cessation of the exercise.

A subset of type $1 \mathrm{DM}$ patients $(\mathrm{N}=22)$ performed cardiac autonomic system evaluation by conventional noninvasive tests: the Valsalva maneuver, beat-to-beat HR variation, the HR response to standing, postural fall in blood pressure, and the sustained handgrip test as previous described $(16,17)$.

\section{Laboratory methods}

Urinary albumin excretion rate (UAER) was measured in 24-h sterile urine samples by immunoturbidimetry in at least two samples collected over the preceding 6 months (MicroAlb Sera-Pak ${ }^{\circledR}$ immuno microalbuminuria; Roche ${ }^{\circledR}$, USA). The results are reported in $\mu \mathrm{g} / \mathrm{min}$ and the mean intra-assay and interassay variation coefficients are 4.5 and $7.6 \%$, respectively. Glycated hemoglobin (A1c test) was measured by high-performance liquid chromatography (reference range 4.7-6.0\%; Merck-Hitachi 9100, Germany). Fasting plasma glucose was measured by the glucose-peroxidase colorimetric enzymatic method (Biodiagnostica, Brazil). Creatinine was measured by the Jaffé method and serum total cholesterol and triglycerides were measured by enzymatic-colorimetric methods (Merck Diagnostica; Boeringher Mannheim, Germany), HDL cholesterol by homogeneous direct method (autoanalyzer, ADVIA 1650; Siemens Healthcare Diagnostics, Germany). LDL cholesterol was calculated using the Friedewald formula (18).

\section{Statistical analysis}

The Student $t$-test or the chi-square test were used to compare clinical and laboratory data. Quantitative variables not having a normal distribution were log transformed. Correlations were performed using the Pearson's chi-square or Spearman's rank correlation depending on the distribution of variables. Multiple logistic regression models were constructed with diabetic retinopathy as the dependent variable, adjusted for A1c test, DM duration, systolic BP, age, UAER, and METs. In each model, one 
variable of HR profile was included: HR recovery at 2 min, $\mathrm{HR}$ recovery at 4 min or maximum increase in HR. The HR variables in the multiple analyses were categorized at intervals of $10 \mathrm{bpm}$ each. Data are reported as mean $\pm S D$, except for triglycerides and UAER, which are reported as median (range). $\mathrm{P}<0.05$ (two-tailed) were considered to be significant.

\section{Results}

Seventy-two type 2 DM patients and 40 type 1 were included. Clinical and laboratory characteristics of the patients are presented in Table 1. Among type 2 DM patients, $22(30.5 \%)$ had some degree of diabetic retinopathy. Patients with diabetic retinopathy had higher A1c values and office BP levels than those without diabetic retinopathy. DM duration, lipid profile, UAER, body mass index, waist circumference, and smoking habit were not different between groups. Among type $1 \mathrm{DM}$ patients, 21 $(52.3 \%)$ had some degree of diabetic retinopathy. Patients with diabetic retinopathy were older than those without. BP levels, glycemic control, DM duration, lipid profile, UAER, body mass index, waist circumference, and smoking habit did not differ between groups.

In type $2 \mathrm{DM}$ patients, resting HR was higher in the patients with diabetic retinopathy, but the HR increase during exercise was lower than in those without diabetic retinopathy (Table 2). The HR recovery at 1 and 2 min after exercise was similar between groups, while at 4 min after exercise the diabetic retinopathy group had lower HR recovery than the group without diabetic retinopathy. The peak systolic and diastolic BP levels during exercise did not differ between groups nor did the maximum BP increment. The systolic BP recovery 2 min after exercise was higher in patients with diabetic retinopathy but diastolic BP recovery was not different between patients with and without diabetic retinopathy.

There was no difference in MET levels achieved in each group $(8.0 \pm 2.1$ vs $8.2 \pm 2 ; P=0.61)$ and all patients reached at least $85 \%$ of the maximal estimated HR frequency.

Based on multivariate logistic regression analysis, increased resting $H R$, lower HR increase and slower HR recovery at 2 and $4 \mathrm{~min}$ were associated with diabetic retinopathy (Table 3 ) before and after adjustment for A1c test, DM duration, systolic BP, age, albuminuria, and METs.

The same pattern of altered HR variability during exercise observed in type $2 \mathrm{DM}$ patients with diabetic retinopathy was observed in type $1 \mathrm{DM}$ : the $\mathrm{HR}$ increase during exercise was greater in the group without diabetic retinopathy (100 \pm 22 vs $79 \pm 16$ bpm; $P=0.001$; Table 2$)$. At 2 min after exercise, HR recovery was lower in patients with diabetic retinopathy ( $47 \pm 26$ vs $62 \pm 15$ bpm; $P=0.03$ ) than in those without diabetic retinopathy. The same pat-

Table 1. Clinical and laboratory characteristics as a function of the presence of diabetic retinopathy in type 2 and type 1 diabetic patients.

\begin{tabular}{|c|c|c|c|c|}
\hline & \multicolumn{2}{|c|}{ Type 2 diabetes retinopathy } & \multicolumn{2}{|c|}{ Type 1 diabetes retinopathy } \\
\hline & Absent $(N=50)$ & Present $(\mathrm{N}=22)$ & Absent $(\mathrm{N}=19)$ & Present $(\mathrm{N}=21)$ \\
\hline Male subjects $(\mathrm{N})$ & $27(54 \%)$ & $13(59 \%)$ & $10(53 \%)$ & $14(67 \%)$ \\
\hline Age (years) & $55.8 \pm 9$ & $53.7 \pm 9$ & $39.5 \pm 10$ & $50.4 \pm 8.5^{\star}$ \\
\hline Diabetes duration (years) & $8.7 \pm 6.8$ & $10.7 \pm 6.0$ & $21.5 \pm 11.7$ & $27 \pm 8.9$ \\
\hline Body mass index $\left(\mathrm{kg} / \mathrm{m}^{2}\right)$ & $28.6 \pm 4.1$ & $29.6 \pm 4.4$ & $24.3 \pm 4.0$ & $23.8 \pm 3.9$ \\
\hline Waist circumference $(\mathrm{m})$ & $0.97 \pm 0.1$ & $0.98 \pm 0.1$ & $86.5 \pm 9.7$ & $83.6 \pm 11.6$ \\
\hline Smoking (N) & $25(50 \%)$ & $8(36 \%)$ & $2(11 \%)$ & $3(14 \%)$ \\
\hline Systolic blood pressure $(\mathrm{mmHg})$ & $130 \pm 15$ & $138 \pm 11^{*}$ & $115 \pm 19$ & $123 \pm 11$ \\
\hline Diastolic blood pressure $(\mathrm{mmHg})$ & $78 \pm 9$ & $83 \pm 7^{*}$ & $75 \pm 12$ & $78 \pm 7$ \\
\hline Glycated hemoglobin (\%) & $6.7 \pm 0.9$ & $8.2 \pm 1.5^{*}$ & $7.5 \pm 1$ & $8.2 \pm 1.4$ \\
\hline Fasting plasma glucose (mg/dL) & $133 \pm 43$ & $165 \pm 50^{*}$ & $154 \pm 68$ & $185 \pm 105$ \\
\hline Total cholesterol (mg/dL) & $195 \pm 31$ & $186 \pm 44$ & $179 \pm 23$ & $171 \pm 32$ \\
\hline High density cholesterol (mg/dL) & $51 \pm 16$ & $47 \pm 11$ & $52 \pm 12$ & $52 \pm 13$ \\
\hline Low density cholesterol (mg/dL) & $115 \pm 29$ & $106 \pm 35$ & $104 \pm 20$ & $100 \pm 20$ \\
\hline Triglycerides $(\mathrm{mg} / \mathrm{dL})$ & $129(39-974)$ & $152(50-555)$ & $83(42-348)$ & $83(32-534)$ \\
\hline Urinary albumin excretion rate ( $\mu \mathrm{g} / \mathrm{min})$ & $8(0.1-1004)$ & $14(0.11-810)$ & $6(0.3-45)$ & $9(0.1-501)$ \\
\hline Creatinine (mg/dL) & $0.8 \pm 0.2$ & $0.7 \pm 0.2$ & $1.0 \pm 0.2$ & $1.2 \pm 0.7$ \\
\hline
\end{tabular}

Data are reported as means \pm SD or number (\%) except for triglycerides and urinary albumin excretion rate, which are reported as median (range). ${ }^{*} \mathrm{P}<0.05$ compared to absence of retinopathy (Student $t$-test for continuous variable; chi-square test for categorical variables). 
tern was observed at 4 min after exercise (60 \pm 20 vs $77 \pm$ $14 \mathrm{bpm} ; \mathrm{P}=0.005)$. The BP parameters analyzed during exercise were similar between groups. Also, lower HR increase during exercise and slower HR recovery at $2 \mathrm{~min}$ were associated with diabetic retinopathy adjusted for DM duration, A1c test and diastolic BP (Table 3).

The parameters of HR changes during exercise correlated with conventional autonomic cardiovascular tests in type $1 \mathrm{DM}$ patients. The mean HR variability during Valsalva maneuver was $2.4 \pm 2.8 \mathrm{bpm}$ and during standing was $1.34 \pm 0.77 \mathrm{bpm}$. The HR recovery at 2 and $4 \mathrm{~min}$ of exercise correlated with HR variability during Valsalva maneuver (2 min: $r=0.679 ; P=0.005 ; 4 \min : r=0.637 ; P$ $=0.01$ ). The $H R$ recovery at $4 \mathrm{~min}$ and $\mathrm{HR}$ maximum increase during exercise correlated with $\mathrm{HR}$ variability in standing tests (HR at 4 min: $r=0.653 ; P=0.01 ; H R$ increment: $r=0.561 ; P=0.03$ ).

\section{Discussion}

This study detected an independent association between diabetic retinopathy and high resting HR, low HR increase and recovery related to exercise. An alteration of the autonomic system in patients with diabetic retinopathy

Table 2. Exercise testing variables as a function of the presence of diabetic retinopathy in type 2 and type 1 diabetic patients.

\begin{tabular}{|c|c|c|c|c|}
\hline & \multicolumn{2}{|c|}{ Type 2 diabetes retinopathy } & \multicolumn{2}{|c|}{ Type 1 diabetes retinopathy } \\
\hline & Absent $(\mathrm{N}=50)$ & Present $(\mathrm{N}=22)$ & Absent $(N=19)$ & Present $(\mathrm{N}=21)$ \\
\hline Resting heart rate $(\mathrm{bpm})$ & $82 \pm 12$ & $89 \pm 10^{*}$ & $79 \pm 17$ & $83 \pm 14$ \\
\hline Peak exercise heart rate (bpm) & $159 \pm 15$ & $152 \pm 17$ & $179 \pm 14$ & $162 \pm 15^{*}$ \\
\hline Heart rate increase (bpm) & $77 \pm 17$ & $64 \pm 18^{*}$ & $100 \pm 22$ & $79 \pm 16^{*}$ \\
\hline Heart rate recovery at $1 \mathrm{~min}(\mathrm{bpm})$ & $13 \pm 11$ & $11 \pm 10$ & $11 \pm 16$ & $14 \pm 17$ \\
\hline Heart rate recovery at $2 \min (\mathrm{bpm})$ & $49 \pm 11$ & $42 \pm 15$ & $62 \pm 15$ & $47 \pm 26^{*}$ \\
\hline Heart rate recovery at $4 \mathrm{~min}(\mathrm{bpm})$ & $60 \pm 12$ & $53 \pm 14^{*}$ & $77 \pm 14$ & $60 \pm 20^{*}$ \\
\hline Resting systolic blood pressure $(\mathrm{mmHg})$ & $130 \pm 15$ & $138 \pm 11^{*}$ & $115 \pm 19$ & $123 \pm 11$ \\
\hline Resting diastolic blood pressure $(\mathrm{mmHg})$ & $78 \pm 9$ & $83 \pm 7^{*}$ & $75 \pm 12$ & $78 \pm 7$ \\
\hline Peak systolic blood pressure $(\mathrm{mmHg})$ & $177 \pm 21$ & $182 \pm 25$ & $170 \pm 31$ & $171 \pm 24$ \\
\hline Peak diastolic blood pressure $(\mathrm{mmHg})$ & $79 \pm 8$ & $83 \pm 13$ & $76 \pm 16$ & $73 \pm 9$ \\
\hline Systolic blood pressure increase $(\mathrm{mmHg})$ & $47 \pm 18$ & $55 \pm 20$ & $54 \pm 26$ & $48 \pm 22$ \\
\hline Diastolic blood pressure increase $(\mathrm{mmHg})$ & $0.4 \pm 7$ & $0.5 \pm 11$ & $1.2 \pm 10$ & $-4 \pm 9$ \\
\hline Systolic blood pressure recovery at $2 \mathrm{~min}$ & $14 \pm 23$ & $24 \pm 16^{*}$ & $9 \pm 15$ & $7 \pm 17$ \\
\hline Diastolic blood pressure recovery at $2 \mathrm{~min}$ & $0.6 \pm 8$ & $0.7 \pm 7$ & $-2 \pm 9$ & $-0.4 \pm 6$ \\
\hline Total metabolic equivalent & $8.2 \pm 2$ & $8 \pm 2.1$ & $11.2 \pm 3$ & $10 \pm 2.3$ \\
\hline
\end{tabular}

Data are reported as means $\pm S D$. Heart rate recovery = maximum heart rate minus heart rate at 1,2 , and 4 min after exercise cessation. Blood pressure recovery $=$ maximum blood pressure level minus blood pressure at 2 nd min after exercise cessation. ${ }^{*} \mathrm{P} \leq$ 0.05 compared to absence of retinopathy (Student $t$-test).

Table 3. Multivariate analysis of heart rate at stress test and the presence of diabetic retinopathy in type 2 and type 1 diabetes.

\begin{tabular}{|c|c|c|c|c|c|c|}
\hline & \multicolumn{3}{|c|}{ Type 2 diabetes retinopathy ${ }^{a}$} & \multicolumn{3}{|c|}{ Type 1 diabetes retinopathy ${ }^{b}$} \\
\hline & OR & $95 \% \mathrm{Cl}$ & $\mathrm{P}$ & OR & $95 \% \mathrm{Cl}$ & $P$ \\
\hline Resting heart rate (bpm) & 1.1 & $1-1.1$ & 0.005 & 1.0 & $0.96-1.05$ & 0.69 \\
\hline Peak exercise heart rate (bpm) & 0.95 & $0.9-1$ & 0.07 & 0.9 & $0.85-0.98$ & 0.01 \\
\hline Heart rate increase (bpm) & 0.90 & $0.85-0.96$ & 0.002 & 0.9 & $0.9-0.99$ & 0.02 \\
\hline Heart rate recovery at $1 \mathrm{~min}(\mathrm{bpm})$ & 0.98 & $0.93-1.0$ & 0.62 & 1.02 & $0.98-1.07$ & 0.26 \\
\hline Heart rate recovery at $2 \mathrm{~min}(\mathrm{bpm})$ & 0.93 & $0.88-0.99$ & 0.02 & 0.96 & $0.92-1.00$ & 0.07 \\
\hline Heart rate recovery at $4 \mathrm{~min}(\mathrm{bpm})$ & 0.91 & $0.85-0.97$ & 0.006 & 0.93 & $0.88-0.99$ & 0.02 \\
\hline
\end{tabular}

aAdjusted for glycated hemoglobin A1c test, diabetes mellitus duration, systolic blood pressure, age, albuminuria, and metabolic equivalents (METs). bAdjusted for glycated hemoglobin A1c test, diabetes mellitus duration and diastolic blood pressure. Heart rate recovery $=$ maximum heart rate minus heart rate at 1,2 , and 4 min after exercise cessation. 
may explain these data as suggested by correlation of these HR changes with cardiovascular autonomic test variables.

The association between diabetic retinopathy and autonomic dysfunction has been suggested by previous studies that evaluated the autonomic system by the cardiovascular tests described by Ewing et al. (16). Krolewski et al. (3), in a cohort of type $1 \mathrm{DM}$ patients followed for 15 to 21 years, showed a relationship between abnormal cardiac autonomic tests and proliferative diabetic retinopathy. Moreover, abnormalities in autonomic ocular function were associated with the development of diabetic retinopathy in a prospective study of 335 type 1 DM patients (19). In type $2 \mathrm{DM}$, autonomic cardiac neuropathy as assessed by $\mathrm{R}-\mathrm{R}$ interval variability and resting $\mathrm{HR}$ was also associated with the severity of diabetic retinopathy $(4,20)$. Different from the present study, these studies included patients with the clinical manifestations of autonomic dysfunction. Moreover, patients also had ischemic heart disease that could lead to alterations in autonomic function (21). In the present study, the patients included did not have ischemic heart disease or clinical manifestation of autonomic neuropathy, suggesting that alterations in HR profile associated with exercise occur earlier than clinically evident autonomic dysfunction.

The evaluation of HR changes related to exercise is a feasible and simple test to perform and has a rational basis to evaluate the autonomic system. During exercise, a combination of increased sympathetic activity and vagal withdrawal increases $\mathrm{HR}$ and the arterial baroreflex is reset in relation to workload $(6,22)$. These coordinated changes in the autonomic system are responsible for the changes in BP and HR during and after exercise. HR recovery after exercise testing is correlated with $H R$ variability in healthy elderly men (23) and acetylcholinesterase inhibition improves HR recovery after exercise in heart failure patients (24), suggesting that the HR profile during exercise could be an early marker of decreased vagal cardiac tonus. This premise is reinforced by data from the present study that showed a strongly significant $(r=0.679$, $P=0.005)$ correlation of $H R$ recovery after exercise with $\mathrm{HR}$ variability on cardiovascular autonomic tests in patients with type $1 \mathrm{DM}$.

One possible explanation is that autonomic dysfunction might be related to diabetic retinopathy pathogenesis through alterations in the BP pattern. We have reported that alterations in BP pattern in type $1 \mathrm{DM}$ patients were associated with diabetic retinopathy (2), and a blunt fall in nocturnal diastolic BP was related to higher sympathetic activity $(17,25)$. These data suggest that alterations in BP pattern are associated with autonomic dysfunction. Moreover, autonomic dysfunction may affect the autoregulation of retina vessels, allowing an abnormal BP pattern or small elevations to have a deleterious impact on the retina.

A possible limitation of this study could be attributed to its cross-sectional design that precludes a causal relationship between the HR exercise profile and diabetic retinopathy development.

Early autonomic dysfunction, assessed by HR changes related to exercise, was associated with diabetic retinopathy. The recognition of HR changes during exercise, a feasible and simple index, might be a practical tool to identify a high-risk group for the presence of diabetic retinopathy.

\section{References}

1. UK Prospective Diabetes Study Group. Tight blood pressure control and risk of macrovascular and microvascular complications in type 2 diabetes: UKPDS 38. BMJ 1998; 317: 703-713.

2. da Costa Rodrigues T, Pecis M, Azevedo MJ, Esteves JF, Gross JL. Ambulatory blood pressure monitoring and progression of retinopathy in normotensive, normoalbuminuric type 1 diabetic patients: a 6-year follow-up study. Diabetes Res Clin Pract 2006; 74: 135-140.

3. Krolewski AS, Barzilay J, Warram JH, Martin BC, Pfeifer M, Rand LI. Risk of early-onset proliferative retinopathy in IDDM is closely related to cardiovascular autonomic neuropathy. Diabetes 1992; 41: 430-437.

4. Smith SE, Smith SA, Brown PM. Cardiac autonomic dysfunction in patients with diabetic retinopathy. Diabetologia 1981; 21: 525-528.

5. Shetler K, Marcus R, Froelicher VF, Vora S, Kalisetti D,
Prakash $\mathrm{M}$, et al. Heart rate recovery: validation and methodologic issues. J Am Coll Cardiol 2001; 38: 1980-1987.

6. Ogoh S, Fisher JP, Dawson EA, White MJ, Secher NH, Raven PB. Autonomic nervous system influence on arterial baroreflex control of heart rate during exercise in humans. $J$ Physiol 2005; 566: 599-611.

7. Cole CR, Blackstone EH, Pashkow FJ, Snader CE, Lauer MS. Heart-rate recovery immediately after exercise as a predictor of mortality. N Engl J Med 1999; 341: 1351-1357.

8. Cole CR, Foody JM, Blackstone EH, Lauer MS. Heart rate recovery after submaximal exercise testing as a predictor of mortality in a cardiovascularly healthy cohort. Ann Intern Med 2000; 132: 552-555.

9. Jouven X, Empana JP, Schwartz PJ, Desnos M, Courbon $D$, Ducimetiere $P$. Heart-rate profile during exercise as a predictor of sudden death. N Engl J Med 2005; 352: 19511958. 
10. Standards of medical care in diabetes - 2007. Diabetes Care 2007; 30 (Suppl 1): S4-S41.

11. Leitao CB, Canani LH, Kramer CK, Boza JC, Pinotti AF, Gross JL. Masked hypertension, urinary albumin excretion rate, and echocardiographic parameters in putatively normotensive type 2 diabetic patients. Diabetes Care 2007; 30: 1255-1260.

12. Canani LH, Gerchman F, Gross JL. Increased familial history of arterial hypertension, coronary heart disease, and renal disease in Brazilian type 2 diabetic patients with diabetic nephropathy. Diabetes Care 1998; 21: 1545-1550.

13. Wilkinson CP, Ferris FL III, Klein RE, Lee PP, Agardh CD, Davis $M$, et al. Proposed international clinical diabetic retinopathy and diabetic macular edema disease severity scales. Ophthalmology 2003; 110: 1677-1682.

14. Boelter MC, Gross JL, Canani LH, Costa LA, Lisboa HR, Tres GS, et al. Proliferative diabetic retinopathy is associated with microalbuminuria in patients with type 2 diabetes. Braz J Med Biol Res 2006; 39: 1033-1039.

15. Fletcher GF, Balady G, Froelicher VF, Hartley LH, Haskell WL, Pollock ML. Exercise standards. A statement for health care professionals from the American Heart Association. Writing Group. Circulation 1995; 91: 580-615.

16. Ewing DJ, Campbell IW, Clarke BF. Assessment of cardiovascular effects in diabetic autonomic neuropathy and prognostic implications. Ann Intern Med 1980; 92: 308-311.

17. Pecis M, Azevedo MJ, Moraes RS, Ferlin EL, Gross JL. Autonomic dysfunction and urinary albumin excretion rate are associated with an abnormal blood pressure pattern in normotensive normoalbuminuric type 1 diabetic patients. Diabetes Care 2000; 23: 989-993.

18. Friedewald WT, Levy RI, Fredrickson DS. Estimation of the concentration of low-density lipoprotein cholesterol in plasma, without use of the preparative ultracentrifuge. Clin Chem 1972; 18: 499-502.

19. Maguire AM, Craig ME, Craighead A, Chan AK, Cusumano $\mathrm{JM}$, Hing SJ, et al. Autonomic nerve testing predicts the development of complications: a 12-year follow-up study. Diabetes Care 2007; 30: 77-82.

20. Imano E, Miyatsuka T, Motomura M, Kanda T, Matsuhisa $\mathrm{M}$, Kajimoto $\mathrm{Y}$, et al. Heart rate elevation and diabetic retinopathy in patients with type 2 diabetes mellitus and normoalbuminuria. Diabetes Res Clin Pract 2001; 52: 185-191.

21. La Rovere MT, Bigger JT Jr, Marcus FI, Mortara A, Schwartz PJ. Baroreflex sensitivity and heart-rate variability in prediction of total cardiac mortality after myocardial infarction. ATRAMI (Autonomic Tone and Reflexes After Myocardial Infarction) Investigators. Lancet 1998; 351: 478-484.

22. Potts JT, Shi XR, Raven PB. Carotid baroreflex responsiveness during dynamic exercise in humans. Am J Physiol 1993; 265: H1928-H1938.

23. Lind L, Andren B. Heart rate recovery after exercise is related to the insulin resistance syndrome and heart rate variability in elderly men. Am Heart J 2002; 144: 666-672.

24. Androne AS, Hryniewicz K, Goldsmith R, Arwady A, Katz $\mathrm{SD}$. Acetylcholinesterase inhibition with pyridostigmine improves heart rate recovery after maximal exercise in patients with chronic heart failure. Heart 2003; 89: 854-858.

25. Nielsen FS, Hansen HP, Jacobsen $P$, Rossing P, Smidt UM, Christensen NJ, et al. Increased sympathetic activity during sleep and nocturnal hypertension in type 2 diabetic patients with diabetic nephropathy. Diabet Med 1999; 16: $555-562$. 